\title{
CONSTRAINED MAGNETIC LEVITATION CONTROL
}

\author{
Mikuláš Huba \& Martin Kamenský \\ Slovak University of Technology in Bratislava, Fac. Electr. Eng. \& Info. Technology, \\ Ilkovičova 3, 81219 Bratislava, Slovakia,huba@elf.stuba.sk, matokam@hotmail.com
}

\begin{abstract}
This paper shows that the highly nonlinear, unstable and fast system of magnetic levitation can be simply controlled by invariant sets based controller generalizing the pole assignment method for constrained systems. This can be reliably tuned by a modification of the well-known step-response based method by Ziegler and Nichols. As all unstable systems, it is tuned in an iterative manner: In the first phase a stabilizing controller is used to measure the static input-output characteristic and to bring the system to a reference starting position for further dynamic experiments, which can be later evaluated to improve the controller structure and tuning. Copyright $($ C) 2005 IFAC
\end{abstract}

Keywords: magnetic bearings, minimum-time pole assignment control, saturation control, nonlinear control systems

\section{INTRODUCTION}

Role of the constraints in the control design becomes to be the central issue of the present control theory. One can find many "academic" solutions tending to guarantee global stability using rigorous and mathematically complex approaches. At the same time, they are very often giving just poor control quality. On the other hand, in practice there are used several ad hoc methods, represented e.g. by the PID tuning proposed by Ziegler and Nichols (1941), which are simply to understand and to use and so popular among the engineering community. The engineering applications naturally do not require global stability, just stability within a working range. Furthermore, in controlling open loop unstable systems with constrained control it is not possible to speak about global stability even under idealized conditions.

The aim of this paper is to show that also in constrained control it is possible to use simple controllers and simple tuning procedures, which can be considered as an extension of the method by Ziegler and Nichols for more demanding applications.

One of the first known applications of magnetic levitation is reported in Alexandria, Egypt, where it was used to rise the sculpture of a goddess. The magnetic force was obviously produced by permanent magnets, so it was not controlled. Because the non-controlled motion in magnetic field is unstable (the force of attraction increases by decreasing the distance from the magnet), the sculpture was supposed to be stabilized by a rope.

Today, magnetic levitation (suspension) systems are often used as a means of eliminating Coulomb friction due to mechanical contact in high-speed transportation systems, magnetic bearings, vibration isolation systems, etc. The force-current-voltage-air gap relationship of the system is unstable and nonlinear, again. The linear approaches rapidly deteriorate with increasing deviations from the nominal operating point. So, alternative solutions based on the gain scheduling (Knospe and Yang, 1997), or the feedback linearization (Trumper, 1997) are used.

Under linear control, the usual way to increase the system robustness by slowing down the transient responses is strongly limited due to the unstable plant dynamics. If the negative feedback established by controller is too week, the levitated ball will remain in one of the limit positions. A higher suppression of disturbances can be achieved by using high gain 
controllers. This, however, leads to problems with the control signal constraints. One way to solve the problem can be represented by the sliding mode control (Lu and Chen, 1995).

This paper treats the possibility of using new type of controllers based on the pole assignment control generalized for constrained systems (Huba, 1999, 2003; Huba and Bisták, 1999; Huba, et al., 1998). Since the adverse effect of the constraints is fully eliminated, it is possible to increase the controller gains up to the level enabled by existing time delays, noise and parameter fluctuations. Such controllers are easy to tune by the step response based method inspired by Ziegler and Nichols.

\section{MAGNETIC LEVITATION SYSTEM}

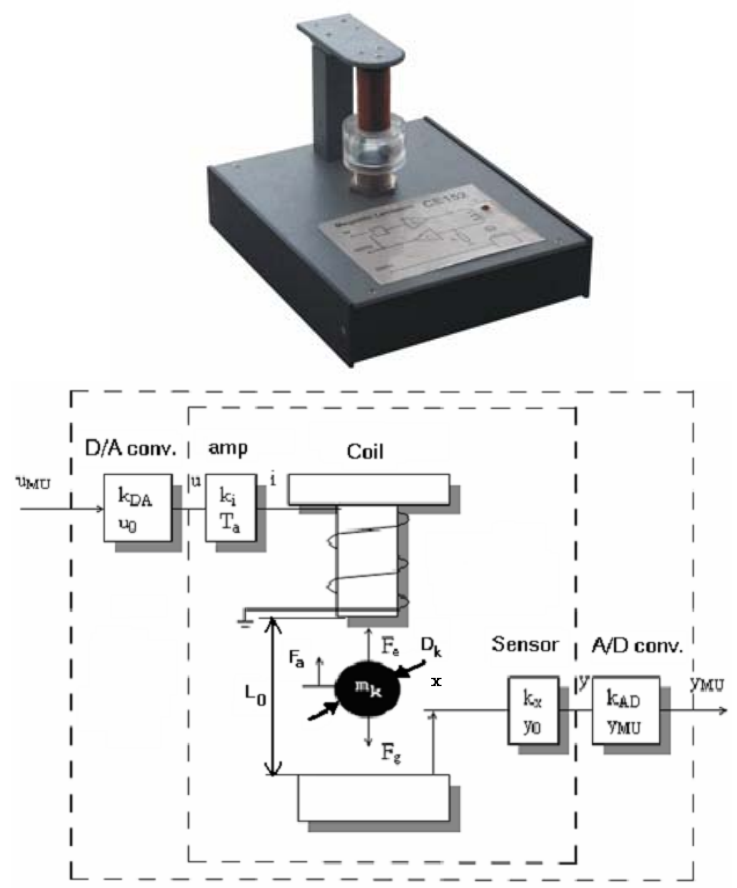

Fig. 1 Magnetic levitation system

The levitated ball (Fig. 1) can be roughly described by the 2 nd order nonlinear equation (Humusoft, 1996a,b).

$$
\begin{array}{ll}
m \ddot{x}+k_{v} \dot{x}=\frac{i^{2} k_{c}}{\left(x-x_{0}\right)^{2}}-m g ; & \left.x \in 0, L_{0}-D_{k}\right\rangle \\
m_{k}=8,37.10^{-3}[\mathrm{~kg}] ; & k_{v}=0,02[\text { N.s.m-1]; } \\
D_{k}=12,7.10^{-3}[\mathrm{~m}] ; & k_{c}=7,4051.10^{-6}[-] ; \\
x_{0}=8,26.10^{-3}[\mathrm{~m}] ; & L_{0}=19.10^{-3}[\mathrm{~m}]
\end{array}
$$

It is controlled via the $\mathrm{D} / \mathrm{A}$ converter described as

$$
u=k_{D A} u_{M U}+u_{00}
$$

$u$ - output converter voltage [V]

$u_{M U}-$ input converter voltage [MU]

$k_{D A}$ - converter gain [V/MU]

$u_{00} \quad$ offset voltage [V]

The ball position is measured using an induction sensor described as

$$
y=k_{x} x+y_{0}
$$

whereby

$k_{x}-$ sensor gain $[\mathrm{V} / \mathrm{m}]$ $y$ - output voltage $[\mathrm{V}]$

$x$ - ball position $[\mathrm{m}]$

$y_{0}$ - offset voltage [V]

The A/D converter can be described as

$$
y_{M U}=k_{A D} y+y_{M U O}
$$

whereby

$y$ - input voltage to converter [V]

$y_{M U}-$ output converter voltage [MU]

$k_{A D}$ - converter gain [V/MU/V]

$y_{M U 0}-$ offset output value $[\mathrm{V}]$

For the given system, following parameters can be identified, which are sometimes different from the values given by producer (in parenthesis):

$$
\begin{array}{ll}
k_{D A}=2.5[\mathrm{~V} / \mathrm{MU}] ; & u_{0}=2.5[\mathrm{~V}] ; \\
k_{A D}=0.2(0.1)[\mathrm{MU} / \mathrm{V}] ; & y_{M U 0}=0[\mathrm{MU}] ; \\
y_{0}=0,0037(0,054)[\mathrm{m}] ; & k_{x}=780,2381 \\
& (797,4603)[\mathrm{V} / \mathrm{m}] ; \\
L_{0}=19.10^{-3}[\mathrm{~m}] &
\end{array}
$$
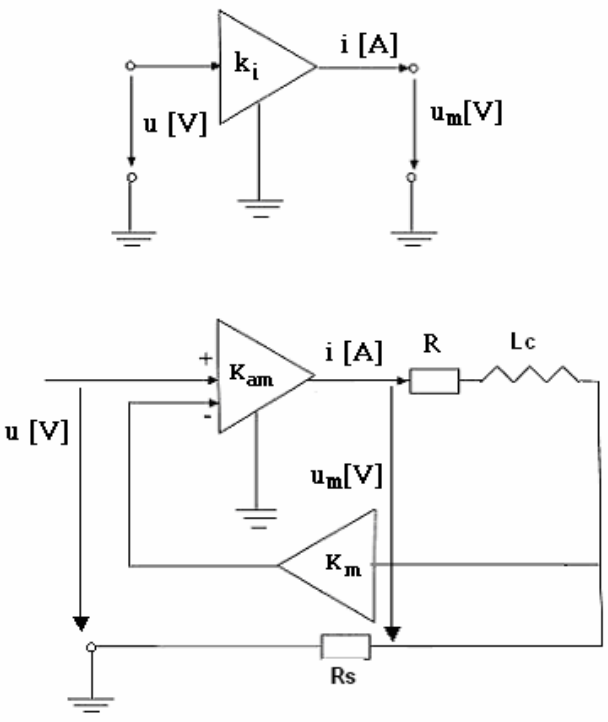

Fig. 2. Electronic amplifier: simplified (above) and detailed scheme (bottom)

The electronic amplifier (Fig. 2) is usually described by equations

$$
\begin{gathered}
u_{m}=i R+\frac{d i}{d t} L_{c} \\
u_{m}=K_{a m}\left(u-R_{s} i\right) \\
\frac{I(s)}{U(s)}=\frac{K_{a m}}{R+K_{a m} K_{m} R_{s}} \frac{1}{\frac{L}{R+K_{a m} K_{m} R_{s}} s+1}= \\
=k_{i} \frac{1}{T_{a} s+1}
\end{gathered}
$$

$K_{a m}$ - amplifier gain

$K_{m} \quad$ - current sensor gain

$R_{s} \quad$ - feedback resistor

$R \quad$ - coil resistor

$L_{c} \quad-$ coil inductance

$k_{i}$ - closed loop amplifier gain

$T_{a}$ - closed loop time constant 
According to the producer (Humusoft, 1996), it is characterized by parameters

$$
\begin{array}{ll}
K_{a m}=100[-] ; & K_{m}=13,33[-] ; \\
R_{s}=0,25[\Omega] ; & R_{c}=3,5[\Omega] ; \\
L_{c}=0,03[\mathrm{H}] ; & k_{i}=0,297[\mathrm{~A} / \mathrm{V}] ; \\
T_{a}=8,9087.10^{-5}[\mathrm{~s}] &
\end{array}
$$

However, records of the input voltage and the (normed) output current (Fig. 3) show that the amplifier dynamics is nonlinear and asymmetric: it depends on the amplitude and polarity of the voltage steps, so that the given value of the time constant $T_{a}$ and the transfer function (7) do not represent reliable information.

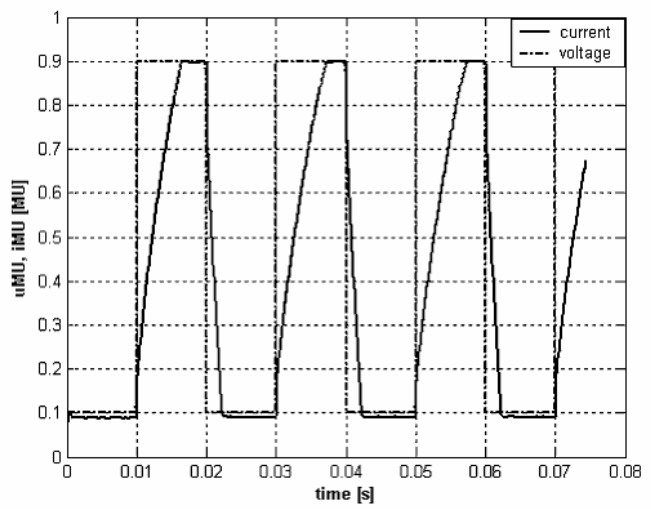

Fig. 3. Current response (normed) to the voltage square signal, $\mathrm{f}=50 \mathrm{~Hz}$

The input-output characteristic of the magnetic levitation system in steady states described by (1-7) should be linear (Fig. 4). The experimentally measured characteristic is, however, reasonably nonlinear. This can be caused by the nonhomogenous magnetic field (Fig. 5). The measured curve was approximated by the $3^{\text {rd }}$ order polynomial, which can be denoted as

$$
y_{M U}=f\left(u_{M U}\right)
$$

Similarly, while the parameter $x_{0}$ (1) should be constant, the evaluation of steady state measurements gives values within the range $<12,20.10^{-3}, 14,7.10^{-3}>$ $m$ (the producer indicates $8,26 \cdot 10^{-3} \mathrm{~m}$ ).

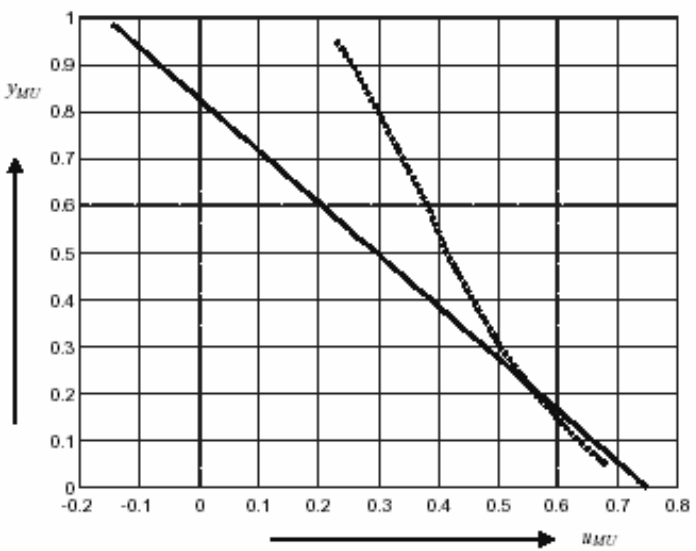

Fig. 4. Input-output behaviour in steady states: Supposed (full curve) and measured (dotted curve)

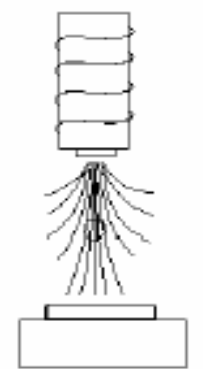

a)

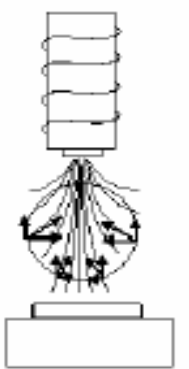

b)
Fig. 5. Possible reason for the mismatch of the supposed and measured input-output behaviour is the non-homogenous magnetic field

All above figures indicate that rigorous control methods based on analytical plant models, as e.g. the feedback linearization method, will yield just limited reliability. Furthermore, the measured signal of the inductive sensor is noisy, whereby the measured noise characteristics - the average value $E(Y)$, the dispersion $D(Y)$ and the standard deviation $S(Y)$ depend on the ball position according to Tabl.1.

Table 1 Measured noise characteristic values

\begin{tabular}{cccc} 
No. & $\mathrm{E}(\mathrm{Y})$ & $\mathrm{D}(\mathrm{Y}) .10^{-7}$ & $\mathrm{~S}(\mathrm{Y}) .10^{-4}$ \\
\hline 1 & $3.68 .10^{-3}$ & 2.26 & 4.76 \\
2 & 0.184 & 2.78 & 5.27 \\
3 & 0.332 & 3.27 & 5.72 \\
4 & 0.573 & 4.75 & 6.89 \\
5 & 0.775 & 6.23 & 7.89 \\
6 & 0.920 & 7.38 & 8.59
\end{tabular}
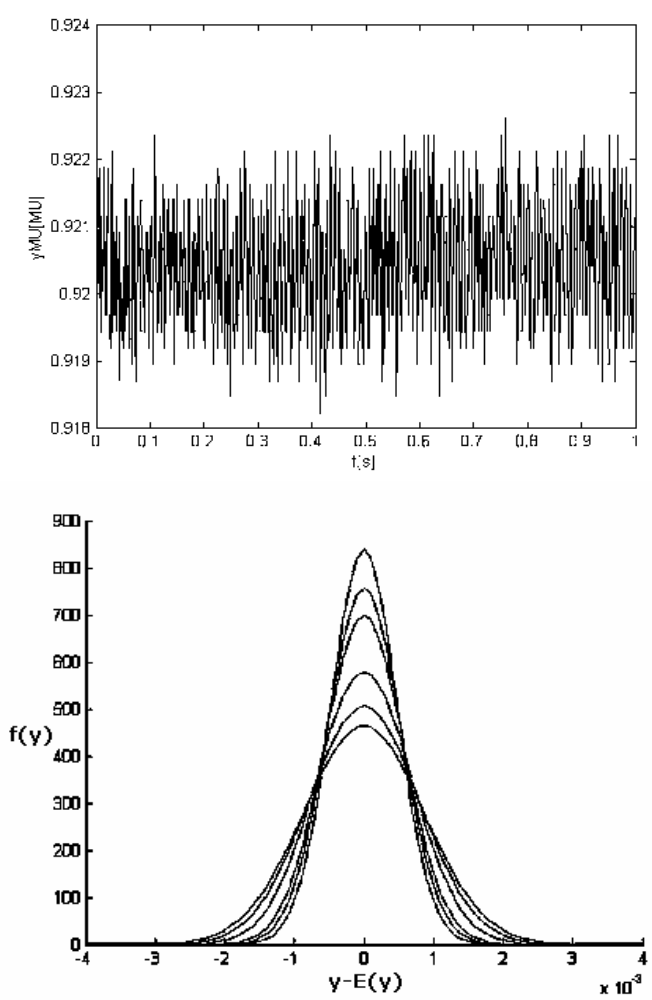

Fig. 6. Noisy measurement corresponding to a fixed ball position (open) and the dispersion of the measured signal, which is growing with the growing ball position (distance from the sensor below) 
Although the maximal noise amplitude is within the range of $0,1-0,2 \%$ of the total measurement range (Fig. 6), by computing the output derivation by means of differentiation $\dot{y}_{M U}=\left(y_{M U k}-y_{M U k-1}\right) / T$ with the sampling periods $T=0,1 \mathrm{~ms}$ the noise amplitudes are within the range 21-33 $y_{M U} / s$. This leads to necessity of additional filtration of the measured output. The best solutions can be achieved by a dual rate approach, when the ball position is measured and the derivation evaluated and filtered with the sampling period $T_{m}=T_{c} / N$, which is just a fraction of the sampling period $T_{c}$ used for control $(N \approx 10)$.

\section{THE DOUBLE INTEGRATOR CONTROL}

The pole assignment controller design is based on requirement of a regular distance decrease of the representative point from the next lower invariant set (Huba, et al., 1999). Being inspired by some problems of the motion control and by the geometrical interpretation of the pole assignment control, the controller (Huba, 1999; Huba and Bisták, 1999; Huba, et al., 1999) is based on specification of an appropriate braking trajectory in the phase plane (invariant set) and on driving representative point toward such trajectory. While for the linear pole assignment control these invariant set (lines, planes, etc.) are traced by the closed loop eigenvectors, for the constrained control they takes more complicated shape. In controlling constrained $2^{\text {nd }}$ order systems, the total transient can be decomposed into a movement along the Reference Braking Curve (RBC - the invariant set of dimension 1) towards the origin (invariant set of dimension 0) and from a general point towards the RBC. Each phase can be characterized by a quotient describing the distance decrease - the closed loop pole. By choosing a particular definition of this distance it is possible to influence the resulting control quality, as well as simplicity of the controller.

When the closed loop poles move along the negative real axis toward infinity in the complex plane, the resulting algorithms correspond to the well-known relay minimum time one. Over an invariant set of linear control surrounding the desired state, the algorithms developed are identical with those of the linear pole assignment control. In difference to many other controllers with variable structure, the proposed solutions represent natural constructions, without any additional parameter, which needs to be tuned.

For the 2nd order systems the controller design consists of following steps:

1. Transformation of the reference state to the origin (invariant set of the dimension 0 ). For a given reference signal $w$ and the real output $y_{r}$ it can be done using equations

$$
\begin{aligned}
& y(t)=y_{r}(t)-w(t) ; \\
& \dot{y}(t)=\dot{y}_{r}(t)-\dot{w}(t) ; \\
& \ddot{y}(t)=\ddot{y}_{r}(t)-\ddot{w}(t)
\end{aligned}
$$

The system description is then

$$
\frac{d \mathbf{x}}{d t}=\mathbf{A}_{c} \mathbf{x}+\mathbf{b}_{c} u ; \quad \mathbf{x}=\left[\begin{array}{ll}
y & \dot{y}
\end{array}\right]^{t}
$$

Its time solution corresponding to the initial state $x_{0}$ and to $u=$ const is

$$
\begin{aligned}
& \mathbf{x}(t)=\mathbf{A}(t) \mathbf{x}_{0}+\mathbf{b}(t) u \\
& \mathbf{A}(t)=e^{\mathbf{A}_{0} t} ; \mathbf{b}(t)=\int_{0}^{t} e^{\mathbf{A}_{0} \tau} \mathbf{b}_{0} d \tau
\end{aligned}
$$

2. For given control signal constraints $u \in U_{1}, U_{2}$ and a chosen closed loop pole $\alpha_{1}$ determination of the line segment of the Reference Braking Curve traced out by the eigenvector with vertices

$$
X_{0}{ }^{j}=\left(\alpha_{1} \mathbf{I}-\mathbf{A}_{0}\right)^{-1} \mathbf{b}_{0} U_{j} ; j=1,2
$$

and determination of the continuation of the RBC corresponding to the limit control values $U_{j}$ with the end at $X_{0}{ }^{j}$ described by equation

$$
X(\tau)^{j}=\mathbf{A}(-\tau) X_{0}^{j}+\mathbf{b}(-\tau) U_{j}
$$

3. Definition of distance $\rho$ of the representative point $\mathbf{x}$ from $\mathrm{RBC}$, e.g. by choosing a direction for its measurement.

4. Deriving the control algorithms decreasing the distance $\rho$ according to

$$
\frac{d \rho}{d t}=\alpha_{2} \rho
$$

with $\alpha_{2}$ being the $2^{\text {nd }}$ closed loop pole.

For the double integrator and the distance measured along the $y$-axis one gets control algorithm, which is far from to be complicated (as usually considered about the constrained control design):

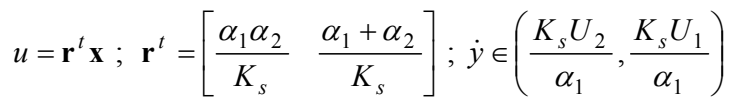

$$
\begin{aligned}
& u=\left[1-\alpha_{2} \frac{y-\frac{1}{2}\left(\frac{\dot{y}^{2}}{K_{s} U_{j}}+\frac{K_{s} U_{j}}{\alpha_{1}^{2}}\right)}{\dot{y}}\right] U_{j} ; \dot{y} \notin\left(\frac{K_{s} U_{2}}{\alpha_{1}}, \frac{K_{s} U_{1}}{\alpha_{1}}\right)
\end{aligned}
$$

Notice that the closed loop properties now depend on the order of the couple of closed loop poles, which enables to design different rates in increasing and decreasing the control signal!

Due to the special properties of the double integrator systems, this algorithm can be used for many linear and nonlinear 2 nd order systems. 


\section{STEP RESPONSE BASED CONTROLLER TUNING}

Papers (Huba, et. al., 1998; Huba, 1999; Huba and Bisták, 1999; Huba, 2003a; Huba, 2003b) show, how it is possible to apply algorithms derived for the double integrator for controlling time delayed and higher order systems by tuning such controllers by the step response based experiments. The controller tuning requires determination of the plant gain $K_{s}$ and of the poles $\alpha_{1,2}$. Both can be determined according to the approximation of the measured process reaction curve by the $\mathrm{I}_{2} \mathrm{~T}_{\mathrm{d}}$-model

$$
S(s)=K_{s} e^{-T_{d} s} / s^{2}
$$

Using the requirement of a triple dominant pole, for this plant it is possible to derive PD-controller coefficients

$$
r_{0}=-0.079 / K_{s} T_{d}^{2} ; r_{l}=-0.461 / K_{s} T_{d}
$$

The same gains can be achieved by setting appropriate poles in (15) denoted as the "equivalent closed loop poles"

$$
\alpha_{1,2}=-(0.23 \pm j 0.16) / T_{d}
$$

If these are approximated by real part (module), one gets real poles

$$
\alpha_{e}=-\frac{0.231}{T_{d}}, \text { or } \alpha_{e}=-\frac{0.282}{T_{d}}
$$

For measurement of the transient responses it is necessary to use stabilizing controller, which can bring the unstable system to a chosen equilibrium point. Since the measurement is noisy, what influences the starting state for the measurement, it is recommended to average several measurements (Fig. 7) and to work with a mean response. The approximation should focus on the initial phase of step responses (Fig. 8).

Using similar procedure for the approximation by the $\mathrm{I}_{2} \mathrm{~T}_{1}$-model

$$
I_{2} T_{1}: \quad S(s)=\frac{K_{s}}{s^{2}\left(T_{1} s+1\right)}
$$

one gets the equivalent poles

$$
\alpha_{1,2}=-(0.167 \pm j 0.096) / T_{1}
$$

These usually lead to slower transients. Because the usual delays represent a mixture of different contributions, the optimal tuning usually lies between these two approximations.

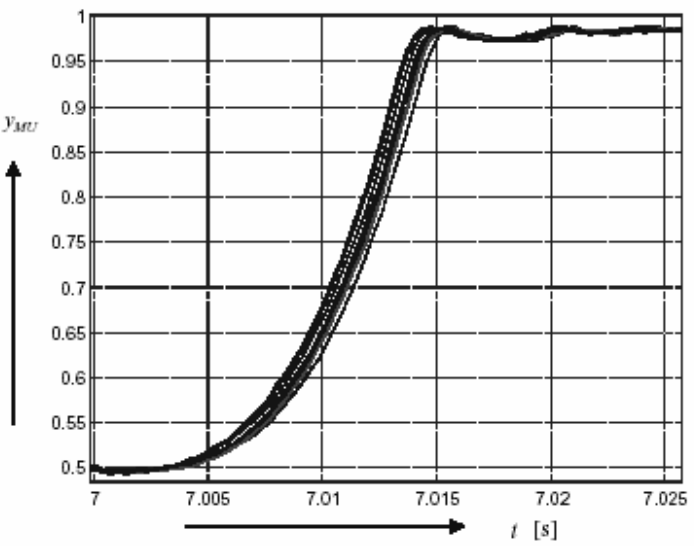

Fig. 7. Measured step responses

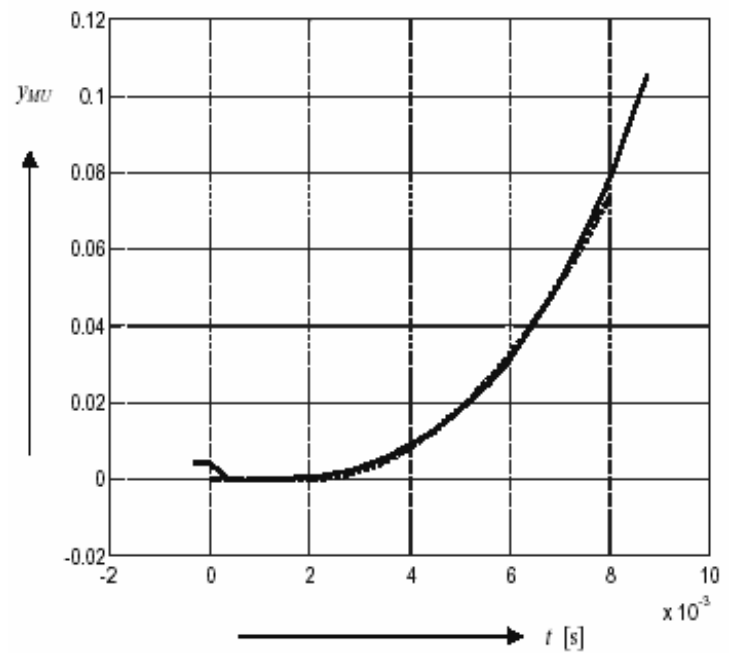

Fig. 8. Step response curve (full curve) and its approximation $\left(T_{d}=2 m s ; K_{s}=7000\right)$

\section{DISTURBATION COMPENSATION}

Since the controller derived for the double integrator control does not consider compensation of constant disturbances (here represented mainly by the gravity force), it should be extended by an additional output term computed by inverse of (8) as

$$
u_{0}=f^{-1}\left(y_{M U}\right)
$$

Instead of $y_{M U}$, also the reference signal $w$ can be used.

Addition of a constant term to the controller output, however, changes the effective control limits $U_{c j} ; j=1,2$ available to controller (Fig. 9).

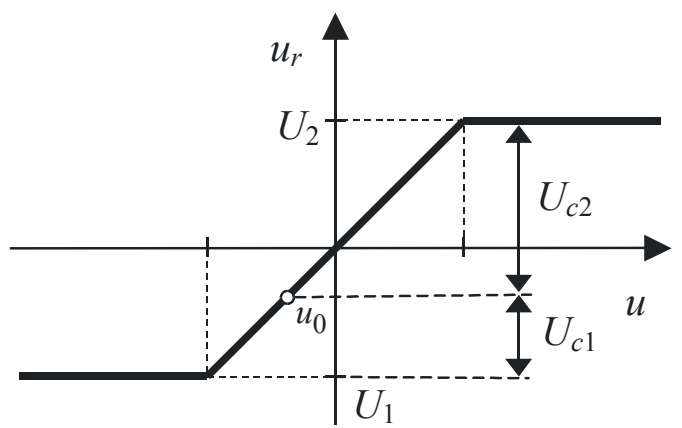

Fig. 9. Transformation of the controller limit values $U_{c j} ; j=1,2$ in the case of a bias $u_{0}$. 
In a steady state (Fig. 10, right) one can write

$$
m_{k} g=K\left(y_{M U}\right) u_{M U}
$$

when

$$
K\left(y_{M U}\right)=\frac{m_{k} g}{u_{M U}}
$$

So, in the steady state the values $y_{M U}$ correspond to those of $u_{M U}$ according to Fig. 4.
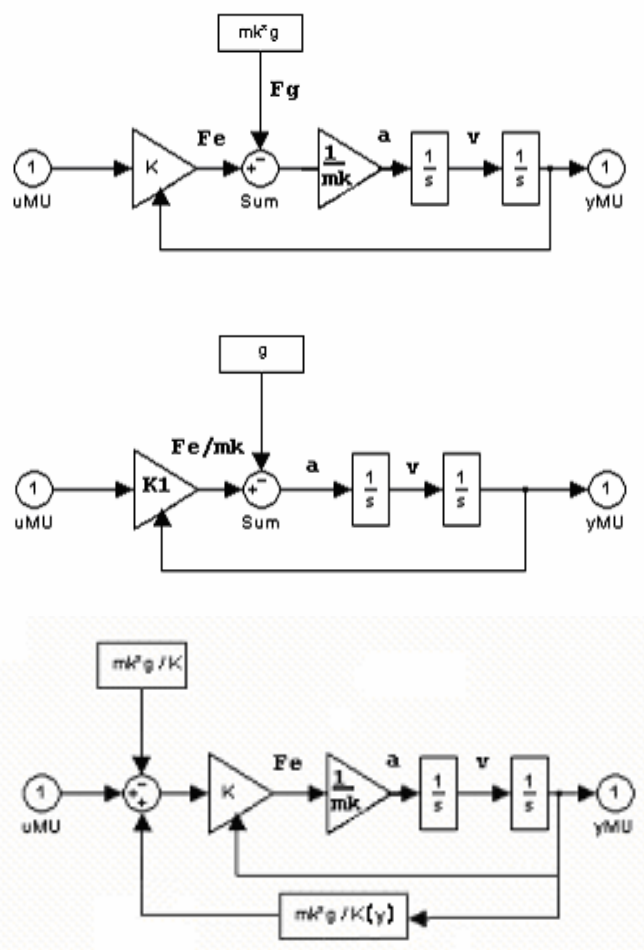

Fig. 10. Two possibilities for modelling the influence of gravitation (above) and compensation of the gravity force by a counteractive signal (below)

\section{EXPERIENTAL RESULTS}

Transient responses (Fig. 11) show high control dynamics without reasonable overshooting. The dynamics can be further improved by testing the system for various steady states and by scheduling the controller parameters.
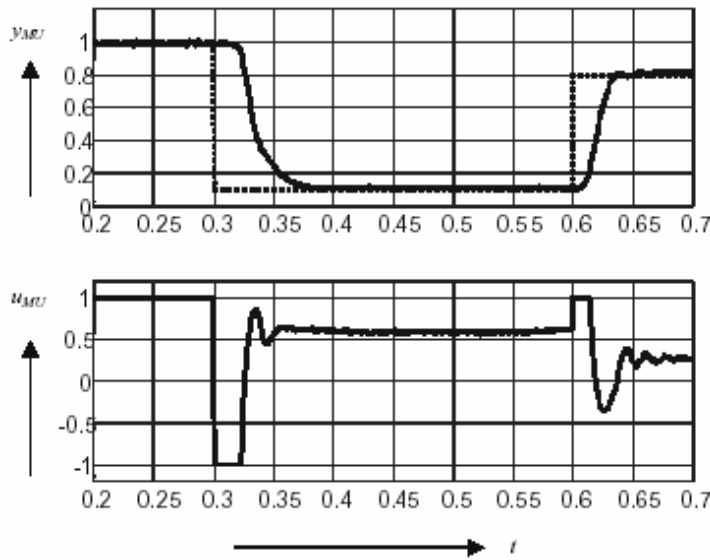

Fig. 11. Transients corresponding to the reference signal (dotted): $T_{d}=2 m s ; K_{s}=7000$

\section{CONCLUSIONS}

It was shown that also a highly nonlinear, unstable and fast system of magnetic levitation may be simply controlled by constrained controller based on the double integrator control. It is reliably tuned by the step response based method interpreted as an extension of the method by Ziegler and Nichols.

\section{ACKNOWLEDGMENTS}

This work has been partially supported by the Slovak Scientific Grant Agency, Grant No. 1/7621/20.

\section{REFERENCES}

Humusoft (1996a). CE 512 Magnetic Levitation Model, User's manual, , Praha

Humusoft (1996b). Educational manual, Praha

Huba,M., Bisták,P., Skachová,Z., Žáková,K. (1998). Predictive Antiwindup PI-and PID-Controllers based on I1 and I2 Models With Dead Time. $6^{\text {th }}$ IEEE Mediterranean Conference on Control and Systems, Alghero.

Huba, M. (1999). Dynamical Classes in the Minimum Time Pole Assignment Control. In: Computing Anticipatory Systems - CASYS '98. Woodbury: American Institute of Physics.

Huba, M. - Bisták, P.: Dynamic Classes in the PID Control. In: Proceedings of the 1999 American Control Conference. San Diego: AACC, 1999.

Huba, M., Sovišová, D. and I. Oravec (1999). Invariant Sets Based Concept of the Pole Assignment Control. In: European Control Conference ECC'99. VDI/VDE Düsseldorf.

Huba, M.( 2003). Constrained systems design. Vol.1 Basic controllers. Vol.2 Basic structures. STU Bratislava (in Slovak).

Knospe, C. and Ch. Yang. (1997). Gain-Schedule Control of a Magnetic Bearing with Low Bias Flux. Proc. $36^{\text {th }}$ IEEE CDC San Diego, CA,

$\mathrm{Lu}, \mathrm{Y}$. and J. Chen (1995). Design of a perturbation estimator using the theory of variable structure systems and its application to magnetic levitation systems, IEEE Trans. On Industrial Electronics, Vol. 42, No. 3, pp. 281-289

Trumper, D. et al.( 1997). Linearizing control of magnetic suspension systems, IEEE Trans. On Control Systems Technology, Vol. 5, No. 4, pp. 427-431

Ziegler, J.G. and N.B. Nichols (1942). Optimum settings for automatic controllers. Trans. ASME, 759-768 